\title{
Bioconvection Unsteady Magnetized Flow in a Horizontal Channel with Dufour and Soret Effects
}

\author{
Muzamil Hussain $\mathbb{D}^{1,2}$ Umer Farooq $\mathbb{D}^{1,3}$ Gulfam Bano, ${ }^{1}$ Jifeng Cui, ${ }^{4}$ \\ and Taseer Muhammad $\mathbb{D D}^{5}$ \\ ${ }^{1}$ Department of Mathematics, COMSATS University Islamabad, Park Road Chak Shahzad, Islamabad 44000, Pakistan \\ ${ }^{2}$ Department of Mathematics, University of the Poonch Rawalakot, Rawalakot 12350, Pakistan \\ ${ }^{3}$ Department of Mathematics, Faculty of Science, Jiangsu University, Zhenjiang 212013, China \\ ${ }^{4}$ College of Science, Inner Mongolia University of Technology, Hohhot 010051, China \\ ${ }^{5}$ Department of Mathematics, College of Sciences, King Khalid University, Abha 61413, Saudi Arabia
}

Correspondence should be addressed to Taseer Muhammad; taseer_qau@yahoo.com

Received 13 July 2021; Revised 8 November 2021; Accepted 14 December 2021; Published 29 January 2022

Academic Editor: Arun K. Saha

Copyright ( 2022 Muzamil Hussain et al. This is an open access article distributed under the Creative Commons Attribution License, which permits unrestricted use, distribution, and reproduction in any medium, provided the original work is properly cited.

\begin{abstract}
In the current decade, bioconvection phenomenon has received a lot of attention in research because of its applications in the biological polymer synthesis, biosensors and biotechnology, pharmaceutical industry, microbial enhanced oil recovery, environmentally friendly applications, and continuous refinements in mathematical modeling. Therefore, this article is prepared to address the unsteady mixed bioconvection in electrically conducting fluid flow between two infinite parallel plates with magnetic field and first-order chemical reaction impacts. Furthermore, the heat and mass transfer study has taken Dufour and Soret effects into account. The nonlinear coupled systems representing the continuity, momentum, energy, mass diffusion, and microorganisms' equations are renewed into an ordinary differential equation (ODE) by employing the similarity renovation. The renovated ODEs are interpreted by the Homotopy Analysis Method (HAM). Impacts of the different emerging parameters, namely, magnetic field parameter $(M)$, heat generation parameter $(Q)$, Dufour number $(D u)$, Soret number $(S r)$, Schmidt number $(S c)$, chemical reaction parameter $\left(K_{0}\right)$, Prandtl number $(P r)$, squeezing parameter $(\beta)$, Peclet number $(P e)$, and Lewis number $(L e)$ on the dimensionless velocity, temperature, concentration, and microorganism profiles as well as the frictional drag, Nusselt number, Sherwood number, and microorganisms mass flux are presented. The main outcomes of this investigation are that the velocity profile rises as the squeezing parameter is increased, and clear enhancement is noticed in the temperature profile for augmented estimations of chemical reaction, heat generation/absorption, and Dufour parameters. There is a significant downward trend in the concentration profile and microorganism density for elevated values of Dufour and Soret parameters.
\end{abstract}

\section{Introduction}

Bioconvection is initiated by the accumulated swimming of motile microorganisms in fluid. This effect happens because microorganisms are slightly denser than water in suspension and usually swim in the upward path. Bioconvection is a growing response due to its use in microfluidic devices, such as bioscience dispersions and bio galvanic devices, and in the investigation of a few thermophilic species existing in hightemperature springs, in microbial oil recovery, and in the formulation of oil and gas transporting sedimentary basins. A relatively new idea of bioconvection prompted by the insertion of microorganisms to a low concentration suspension of nanoparticles has drawn the consideration of researchers. Pal and Mondal [1] reported that bioconvection improves the stability of nanofluid flow. Kuznetsov and Avramenko [2] examined the bioconvection in fluid flow that contained gyrotactic microorganisms and nanoparticles. Khan et al. [3] addressed boundary layer nanofluid flow comprising microorganisms with Naiver slip across a 
vertical plate. Tham et al. [4] researched the bioconvection flow over a solid domain with the effects of gyrotactic microorganism density factor. $\mathrm{Xu}$ et al. [5] analyzed the completely developed flow using nanofluid having both gyrotactic microorganisms and nanoparticles in a horizontally placed channel. Raees et al. [6] studied bioconvective unsteady flow of Newtonian fluid with nanoparticles between two parallel plates. Mosayebidorcheh et al. [7] examined fluid flow including nanoparticles in a horizontal channel with gyrotactic microorganisms. Shen et al. [8] studied analytically bioconvective nanofluid flow carrying motile microorganisms across a stretched surface with radiation and velocity slip impacts by employing HAM. Kumar et al. [9] analyzed the unsteady bioconvective nanofluid flow with slip velocity, thermophoresis, and Brownian effects by employing the Keller-box method. Zhao et al. [10] explored an electrically conducting unsteady mixed bioconvection fluid flow between two plates. Tarakaramu and Satya Narayana [11] investigated the flow of bioconvection nanofluids in a rotating system with binary chemical reaction effects. Waqas et al. [12] visualized nanofluid flow with heat transfer rates and motile microorganisms across a stretching surface. Rashad and Nabwey [13] examined bioconvection and nanofluid flow over a horizontal cylinder. Shukla et al. [14] discussed heat transfer in bioconvective nanofluid flow with solar flux, radiation, and oblique magnetic field impacts.

In the thermochemical process, convection by two distinct rates of diffusion is used in a variety of biomedical applications such as laser tumor therapy, improving oxygenated blood movement, polymeric liquids, and novel lubricants. The Soret (thermal diffusion) effect is the variation in mass flux induced by a temperature difference. However, the Dufour effect is usually defined as the heat flux caused by the concentration gradient. Soret impact is used to manage mixtures of gases with lighter and medium molecular weights. These phenomena have many practical applications in the fields of geoscience, chemical engineering, air pollution, isotope separation, purification of ground water, hydrology, etc. Researchers have paid extensive attention to these two aspects because of the abovementioned applications. Cheng [15] explained natural convection with Soret $(\mathrm{Sr})$ and Dufour $(\mathrm{Du})$ effects on a fluid flow saturated in a porous medium. Hayat et al. [16] investigated viscoelastic fluid flow over a porous surface with a magnetic field, and $D u$ and $S r$ impacts. Hayat and Nawaz [17] also studied $D u$ and $S r$ effects for second-grade fluid flow. Unsteady MHD flow on a radiative porous plate along with binary chemical reaction, and $D u$ and $S r$ effects was reviewed by Sharma et al. [18]. Hayat et al. [19] analyzed the $\mathrm{Sr}$ and $\mathrm{Du}$ impacts on 3-D viscoelastic fluid flow. Moorthy et al. [20] investigated MHD and convection flow across a porous surface with $\mathrm{Du}$ and $\mathrm{Sr}$ impacts. Sheri and Srinivasa Raju [21] examined the Soret effect on the time-dependent MHD fluid flow across a semi-infinite vertical surface with the consideration of viscous dissipation. Majeed et al. [22] explored the $\mathrm{Du}$ and $\mathrm{Sr}$ influences on second-grade fluid flow induced by an expanding cylinder with radiation effects. Liu et al. [23] utilized multirelaxation phenomena to develop a dual-diffusion natural convective flow with $D u$ and $S r$ effects by using lattice Boltzmann theory. They showed that double-diffuse natural convective flow may be easily achieved via the use of the $D u$ and $S r$ impacts. Sardar et al. [24] evaluated mixed convection processes in a Carreau fluid flow with $\mathrm{Du}$ and $\mathrm{Sr}$ influences that were confined by a wedge. Bilal Ashraf et al. [25] deliberated the mixed convective MHD viscoelastic fluid flow with $D u$ and $S r$ impacts. Jiang et al. [26] demonstrated promising results in simultaneous heat and mass transfer processes with $\mathrm{Du}$ and $\mathrm{Sr}$ impacts. Hafeez et al. [27] studied the fluid flow over a disk with thermophoresis $\mathrm{Du}$ and $\mathrm{Sr}$ effects.

Hannes Alfvén, in 1970, was the first person who introduced and developed Magnetohydrodynamics (MHD). MHD is a dynamics study in the presence of electrically conducting liquids with magnetic properties and its effects that has sufficient applications in biomedical sciences and engineering such as drug targeting, biowaste fluid transportation, cell separation, cancer tumor treatment, magnetic endoscopy, astrophysics, MHD pumps, metallurgy, ship propulsion, reduction of turbulent drag, jet printers, and fusion reactors. MHD flow across different geometries relevant to engineering is an attractive and appreciable field of science. The above-mentioned applications of MHD compel scientists to create innovative mathematical models in the fluid mechanics field [28-31]. Patel and Singh [32] analyzed the MHD, micropolar fluid flow with Brownian diffusion, and convective boundary condition. Aly and Pop [33] explored steady MHD hybrid nanofluid flow along the permeable flat plate. Rashid et al. [34] studied the MHD boundary layer flow over a porous shrinking surface with the radiation effects. Waini et al. [35] evaluated the steady fluid flow across a permeable wedge with magnetic field impacts. Naqvi et al. [36] addressed the chemical reaction and radiation impacts in MHD nanofluid flow over a radially stretching/shrinking disk. Raees et al. [37] examined mixed convection in a magnetized second-grade fluid flow over a stretched surface. Rizwana et al. [38] investigated MHD stagnation fluid flow with mixed convection across an oscillating plate.

The motivation of the current study is to examine the unsteady bioconvective flow in a horizontal squeezing channel. Multiple results of the magnetic field, chemical reaction, and Dufour and Soret effects are employed into the account. The considered system of PDEs is transformed into the dimensionless ODEs by employing the similarity transformation Then, these converted ODEs are solved by adopting HAM (introduced by Liao [39]). We have successfully implemented HAM to solve the dimensionless form of momentum, energy, nanoparticles mass, and bioconvection (motile microorganism species) equations with suitable boundary conditions. Extensive graphical and tabulated results are presented with the aid of Mathematica software, and finally, the impacts of different emerging parameters on the velocity, temperature, nanoparticles fraction, and motile microorganism profiles along with the frictional drag, Nusselt number, Sherwood number, and microorganisms mass flux are examined and deliberated in detail. This research may be helpful for researchers and 
engineers who work on the industrial applications of bioconvection squeezed flow of nanofluid which is quite useful in the fields of polymer synthesis, biomedicine, lubrication, metal and polymer molding, foam production process geothermal system, and many others.

\section{Mathematical Formulation}

MHD and unsteady electrically conducting bioconvective nanofluid flow between two infinite parallel plates with firstorder chemical reaction, and Dufour and Soret effects are considered. The coordinate system is considered in such a way that the $x$-axis is along the lower plate and $y$-axis is normal to the flow direction. Figure 1 depicts the geometry of the flow system. The plates are assumed at $y=h(t)=$ $[\nu(1-a t) / b]^{1 / 2}$ distance apart, and the top plate is assumed to move in towards or away directions of the lower plate with velocity $v(t)=d h / d t$. Here, $t$ denotes the time, $v$ is the kinetic viscosity, and $a, b$ are the positive numbers. Definitely, it is clear that $1-a t>0$, which shows that $a<\sqrt{b^{2}-4 a c} 1 / t$. Clearly, $a=0$ indicates that both plates are static, $0<a<1 / t$ illustrates that the upper plate is squeezed against the lower plate, and $a<0$ demonstrates that the upper plate is gone away from the lower one. Also, it is assumed that the upper and lower plates are kept at a constant chemical reaction concentration $C_{2}$ and $C_{1}$; constant temperatures and constant microorganisms' concentration are $T_{2}, T_{1} N_{2}$, and $N_{1}$, respectively. The magnetic field $(t)$ is considered along the $y$-axis.

The governing system under the above-mentioned assumptions are

$$
\nabla . V=0,
$$

$$
\frac{\partial V}{\partial t}+(V . \nabla) V=v \nabla^{2} V+\frac{1}{\rho}(J \times B)-\frac{1}{\rho} \nabla p,
$$

$$
\begin{aligned}
\frac{\partial T}{\partial t}+V \cdot \nabla T & =\alpha \nabla^{2} T+\frac{Q_{s}(t)}{\rho C_{p}}\left(T-T_{0}\right)+J, \\
\frac{\partial C}{\partial t}+V \cdot \nabla C & =D \nabla^{2} C-k(t)\left(C-C_{0}\right)+R, \\
\frac{\partial N}{\partial t} & =-\nabla \cdot j .
\end{aligned}
$$

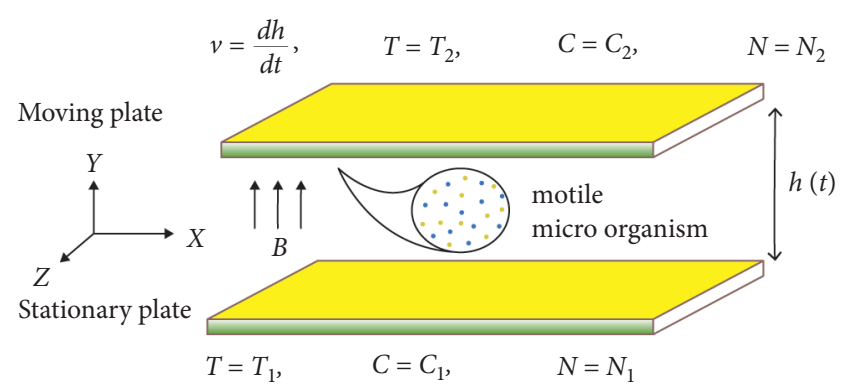

Figure 1: Flow configuration.

where $V, B, J, \rho, p, v, T_{0}, T, \alpha, Q_{s}(t), D, C, C_{0}, K(t), N$, and $j$ are the velocity vector, magnetic induction along $y$-direction, electric current density, fluid density, pressure, kinematic viscosity, reference temperature, temperature, thermal diffusivity, volumetric heat generation rate, mass diffusivity, chemical reaction, reference concentrations, reaction rate, motile microorganism concentration, and flux of microorganisms, respectively.

$J$ is the heat flux, known as the "Dufour effect," that can be given by Frick's law of diffusion as $J=\rho D K_{t} / C_{s} \nabla^{2} C, R$ is the flux concentration generated by the temperature difference known as "Soret effect," from Fourier's law of heat conduction $R_{c}=q / A=D K_{t} / T_{m} \nabla^{2} T . T_{m}, D$, and $K_{t}$ are the mean temperature, coefficient of diffusion species, and thermal diffusion ratio, respectively.

Momentum Equation (2) in the component form of a two-dimensional channel flow can be written as

$$
\begin{aligned}
& \frac{\partial u}{\partial t}+u \frac{\partial u}{\partial x}+v \frac{\partial u}{\partial y}=-\frac{\partial p}{\rho \partial x}+v\left(\frac{\partial^{2} u}{\partial x^{2}}+\frac{\partial^{2} u}{\partial y^{2}}\right)-\frac{\sigma B^{2}(t) u}{\rho} \\
& \frac{\partial v}{\partial t}+u \frac{\partial v}{\partial x}+v \frac{\partial v}{\partial y}=-\frac{\partial p}{\rho \partial y}+v\left(\frac{\partial^{2} v}{\partial x^{2}}+\frac{\partial^{2} v}{\partial y^{2}}\right) .
\end{aligned}
$$

To simplify equations (6) and (7), the given transformation is used:

$$
\xi=\frac{\partial v}{\partial x}-\frac{\partial u}{\partial y}=-\nabla^{2} \psi
$$

By using the above information, the governing equations are transformed as follows:

$$
\begin{gathered}
\frac{\partial u}{\partial x}+\frac{\partial v}{\partial y}=0 \\
\frac{\partial \xi}{\partial t}+u \frac{\partial \xi}{\partial x}+v \frac{\partial \xi}{\partial y}=v\left(\frac{\partial^{2} \xi}{\partial x^{2}}+\frac{\partial^{2} \xi}{\partial y^{2}}\right)+\frac{\sigma B^{2}(t)}{\rho} \frac{\partial u}{\partial y}, \\
\frac{\partial T}{\partial t}+u \frac{\partial T}{\partial x}+v \frac{\partial T}{\partial y}=\frac{k}{\rho C_{p}}\left(\frac{\partial^{2} T}{\partial x^{2}}+\frac{\partial^{2} T}{\partial y^{2}}\right)+\frac{Q_{s}(t)}{\rho C_{p}}\left(T-T_{0}\right)+\frac{D k_{t}}{C_{s} C_{p}}\left(\frac{\partial^{2} C}{\partial x^{2}}+\frac{\partial^{2} C}{\partial y^{2}}\right),
\end{gathered}
$$




$$
\begin{aligned}
& \frac{\partial C}{\partial t}+u \frac{\partial C}{\partial x}+v \frac{\partial C}{\partial y}=D\left(\frac{\partial^{2} C}{\partial x^{2}}+\frac{\partial^{2} C}{\partial y^{2}}\right)-K(t)\left(C-C_{0}\right)+\frac{D k_{t}}{T_{m}}\left(\frac{\partial^{2} T}{\partial x^{2}}+\frac{\partial^{2} T}{\partial y^{2}}\right) \\
& \frac{\partial N}{\partial t}++\frac{\partial}{\partial y}(N \widetilde{v})+u \frac{\partial N}{\partial x}+v \frac{\partial N}{\partial y}+\frac{\partial}{\partial x}(N \widetilde{v})=D_{m}\left(\frac{\partial^{2} N}{\partial x^{2}}+\frac{\partial^{2} N}{\partial y^{2}}\right) .
\end{aligned}
$$

Boundary conditions are as follows:

$$
\begin{aligned}
u & =0, \\
v & =0, \\
T & =T_{1}, \\
C & =C_{1}, \\
N & =N_{1}, \\
\text { at } y & =0, \\
u & =0, \\
v & =\frac{d h}{d t}, \\
T & =T_{2}, \\
C & =C_{2}, \\
N & =N_{2}, \\
\text { as } y & =h(t),
\end{aligned}
$$

where $u=\partial \psi / \partial y$ and $v=-\partial \psi / \partial x$ are the velocity components, $\xi=-\nabla^{2} \psi$ is the vorticity function, $N$ represents motile microorganisms density, $W_{c}$ is the maximum cell speed, $\widetilde{v}=\left[b_{c} W_{c} /\left(C_{1}-C_{0}\right) \partial C / \partial y\right]$ is the microorganisms' average swimming velocity, $b_{c}$ is chemotaxis constant, and $D_{m}$ is the diffusivity of microorganisms.

Similarity transformations are the transformations that can be used to convert an $n$-independent variable partial differential system to a system with $(n-1)$ independent variables. When $n=2$, the situation is ideal since one is dealing with ODEs rather than PDEs. For the solution of nondimensional problem, the following similarity transformations and nondimensional quantities have been used:

$$
\begin{aligned}
\psi(x, y) & =\left(\frac{b v}{1-a t}\right)^{1 / 2} x f(\eta), \\
u & =\frac{b x}{1-a t} f^{\prime}(\eta) \\
v & =-\left(\frac{b v}{1-a t}\right)^{1 / 2} f(\eta), \\
\eta & =\left(\frac{b}{v(1-a t)}\right)^{1 / 2} y, \\
\theta(\eta) & =\frac{T-T_{0}}{T_{1}-T_{0}}, \phi(\eta)=\frac{C-C_{0}}{C_{1}-C_{0}} \\
w(\eta) & =\frac{N}{N_{1}} .
\end{aligned}
$$

Using equation (14) into equations 9-12, we get the following nondimensional equations of momentum, energy, concentration, and microorganisms equations; the continuity equation (10) is satisfied identically as follows:

$$
\begin{gathered}
f^{\prime \prime \prime}-M f^{\prime \prime}-\beta \eta f^{\prime \prime \prime}-3 \beta f^{\prime}-f^{\prime \prime} f^{\prime}+f^{\prime \prime \prime} f=0, \\
\theta \prime \prime+\operatorname{PrQ} \theta-\operatorname{Pr} \beta \eta \theta^{\prime}+\operatorname{Pr} f \theta^{\prime}+D u \operatorname{Pr} \phi^{\prime}=0, \\
\phi^{\prime \prime}-S c K_{0} \phi-S c \phi^{\prime} \beta \eta+S c f \phi^{\prime}+S C S r \theta^{\prime \prime}, \\
w^{\prime \prime}-S c \beta \eta w^{\prime}+S c f w^{\prime}-\operatorname{Pew} \phi^{\prime \prime}-\operatorname{Pe} \phi^{\prime} w^{\prime}=0 .
\end{gathered}
$$

According to equation (13), the transform boundary conditions are

$$
\begin{aligned}
f(0) & =0, \\
f^{\prime}(0) & =0, \\
\theta(0) & =1, \\
(0) & =1, \\
w(0) & =1, \\
f(1) & =\beta, \\
f^{\prime}(1) & =0, \\
\theta(1) & =\delta_{\theta}, \\
(1) & =\delta_{\phi}, \\
w(1) & =\delta_{w},
\end{aligned}
$$

where $\beta=a / 2 b$ is the squeezing parameter, $M=\sigma / v \rho B_{0}^{2}$ is the dimensionless magnetic field number, $\operatorname{Pr}=\rho C_{P} v / k$ indicates the Prandtl number, $Q=Q_{0} / \rho C_{p}$ signifies the heat generation parameter, $D u=D k_{T}\left(C_{1}-C_{0}\right) / v C_{p}\left(T_{1}-T_{0}\right)$ and $S r=D k_{T}\left(T_{1}-T_{0}\right) / v T_{m}\left(C_{1}-C_{0}\right)$ represents the Dufour and Soret numbers, $S c=v / D$ is the Schmidt number, $K_{0}, P e$, and $L e=\alpha / D$ are the chemical reaction parameter, bio convection Peclet number, and Lewis number, respectively, $\delta_{\theta}=T_{2}-T_{0} / T_{1}-T_{0}, \delta_{\phi}=$ $C_{2}-C_{0} / C_{1}-C_{0}$, and $\delta_{w}=N_{2} / N_{1}$ are the constants.where $B_{0}=(b / v(1-a t))^{-3 / 4} B(t)$ and $Q_{0}=(b / 1-a t)^{-1} Q_{s}(t)$ are the reference magnetic field and heat generation, respectively, while $K_{0}=(b / 1-a t)^{-1} k(t)$ is the chemical reaction parameter.

\section{Thermal Transport Analysis}

The nonlinear ODEs (15-19) are tackled by employing HAM. This method is used to find the analytic solutions of the complicated nonlinear ordinary differential system. It has unique advantages as compared to other analytical approximation methods. Besides the other different 


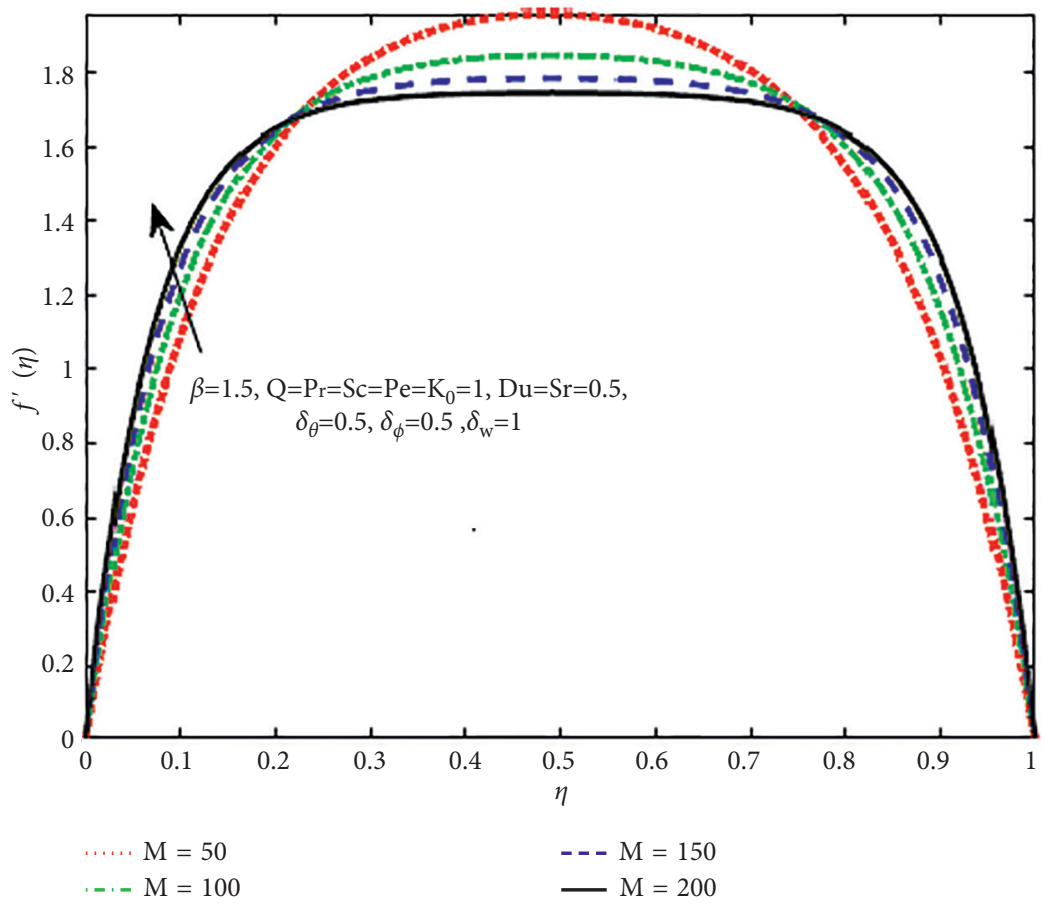

Figure 2: Magnetic parameter (M) effect on $\mathbf{f} \prime(\eta)$.

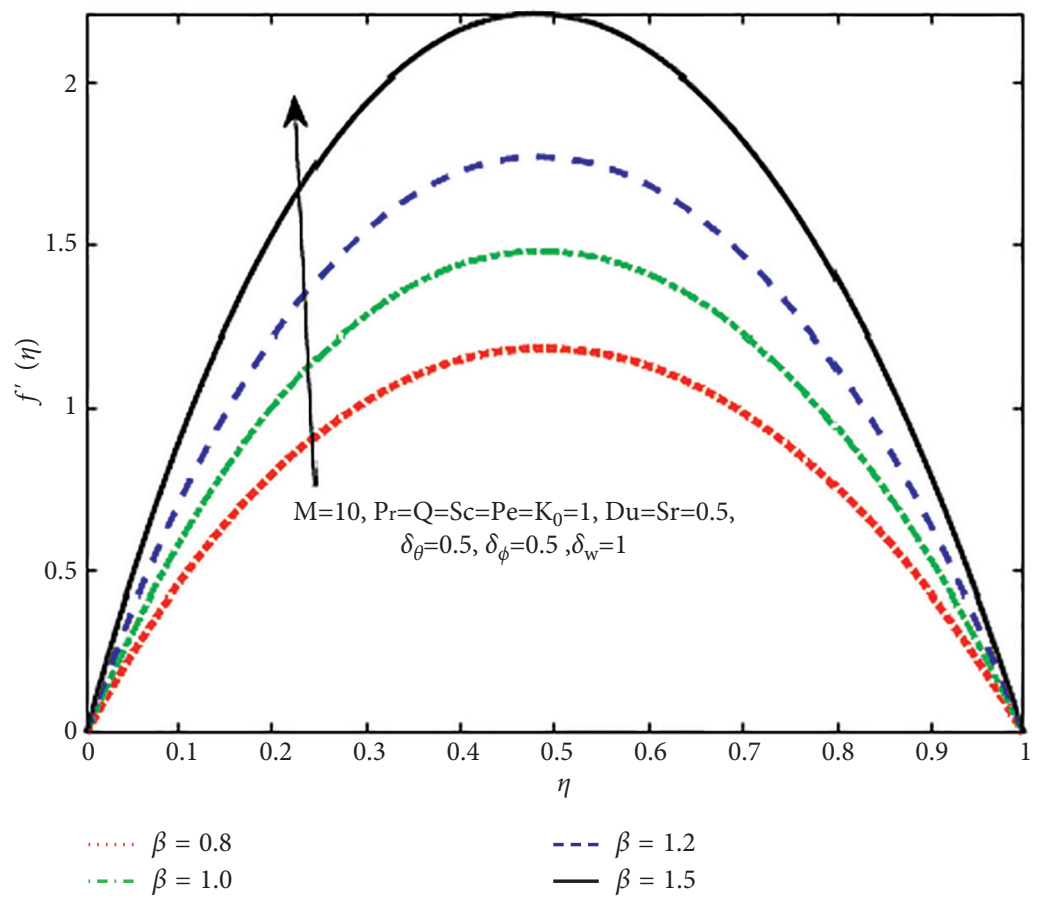

FIGURE 3: Squeezing parameter $(\beta)$ effect on $\mathbf{f} \prime(\eta)$.

analytical techniques, HAM delivers convenient results. It is investigated that the nonlinear boundary value problems (BVPs) of science, finance, and engineering can be analytically solved by applying HAM. It is developed on Mathematica and Maple softwares that can effectively be used on finite, semifinite, and infinite intervals to solve eigen value problems. Convergence of the resulting solution can be found with the help of a module in HAM by using the optimum value called the "convergence-control parameter," which can be used at the minimum squared residual of the system of the governing equations in a certain order of approximations. 


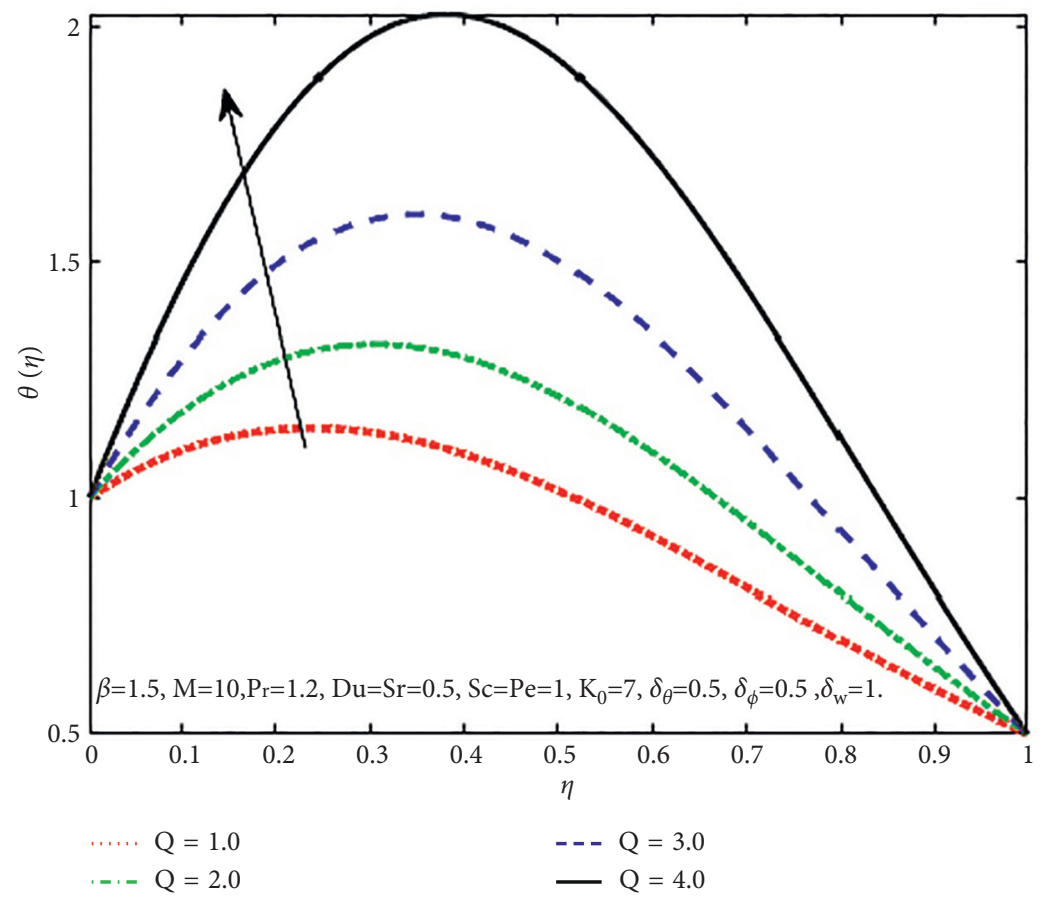

FIgURE 4: Heat generation parameter (Q) effect on $\theta(\eta)$.

Figure 2 portrays the impacts of magnetic parameter $(M)$ on the velocity profile $\left(\mathbf{f}^{\prime}(\eta)\right)$. It is seen that the velocity profile develops rapidly with the evolution of $M$, while the increment in $M$ boosts the flow velocity in the vicinities of lower and upper plates, but decline in the flow velocity is observed between the plates. The fact behind this is that, the magnetic field interacts with the electrical conductivity of nanofluid and formed Lorentz forces which diminish the velocity of fluid. The influence of the squeezing parameter on the velocity profile is observed in Figure 3. It predicts that the velocity profile rises as the squeezing parameter $(\beta)$ increases; this is because that the accelerated flow has a higher velocity. It is seen in Figure 4 that the temperature profile is greatly influenced by heat generation parameter $(Q)$, although continuously increasing $Q$ raises the temperature profile rapidly. Physically, increasing $Q$ increases the kinetic energy of the fluid particles, so the thickness of the thermal boundary layer rises, which leads to an increase in temperature profile. Figure 5 reveals that the temperature profile increases constantly when the chemical reaction parameter $\left(K_{0}\right)$ rises. This occurred because of an increase in the concentration of interfacial nanoparticles.

Figure 6 demonstrates the effect of Dufour number $(D u)$ on the temperature profile. Graph clearly portrays that $D u$ has a great influence on temperature profile. It is noticed that on enhancing $D u$, the thermal profile is strengthened. This can be attributed to an increase in the $D u$, which leads to a rise in the concentration gradient, resulting in rapid mass diffusion. As a result, the rate of energy transfer between particles increases. Consequently, the temperature profiles rise. Figure 7 reveals the comportment of Soret number $(\mathrm{Sr})$ on temperature profile $(\theta(\eta))$. Rising values of $\mathrm{Sr}$ strengthens the thermal field.
Figures 8-12 depict the impacts of heat generation/absorption $(Q)$, chemical reaction parameters $\left(K_{0}\right)$, Dufour $(\mathrm{Du})$, Soret $(\mathrm{Sr})$, and Schmidt $(\mathrm{Sc})$ numbers on concentration profile $(\phi(\eta))$. It is established that these physical parameters have a major impact on concentration. Figure 8 portrays the nanoparticle concentration profile for the heat generation parameter $(Q)$. The figure clearly shows that both the concentration and thickness of the concentration boundary layer are the decreasing functions of $Q$. Figure 9 illustrates the impact of chemical reaction parameter $\left(K_{0}\right)$ on concentration profile $(\phi(\eta))$. It is noted that $\phi(\eta)$ and concentration boundary layer decline by raising the value of $K_{0}$. This decline in $\phi(\eta)$ is due to a decrease in molecular diffusivity as the number of chemical species increases. Figure 10 explores the conduct of Dufour parameter $(\mathrm{Du})$ on the concentration profile of nanoparticles $(\phi(\eta))$. It is clear from this graph that $\phi(\eta)$ decreases for an increasing value of $D u$. Figure 11 explicates the effects of Soret number $(S r)$ on the nanoparticle's concentration profile of $(\phi(\eta))$. It represents a decreasing trend in $\phi(\eta)$ for the uprising values of Sr. Figure 13 inspects the consequences of the Schmidt number $(S c)$ on the concentration field $(\phi(\eta))$. It is observed that by increasing $S c$, the concentration profile decays due to a reduction in mass diffusion.

Figures 13-14 illustrate the effects of Dufour (Du) and Soret ( $\mathrm{Sr}$ ) on microorganism's concentration profile. It is found that increasing estimations of $D u$ and $S r$ numbers reduce the density of microorganism's concentration $(w(\eta))$. The effect of Peclet number $(P e)$ against $w(\eta)$ is plotted in Figure 15. It illustrates that when $P e$ increases, the microorganism density decreases. Physically, $P e$ is a measurement of the relative strength of motile microorganisms' directional and random swimming. So, larger $P e$ values 


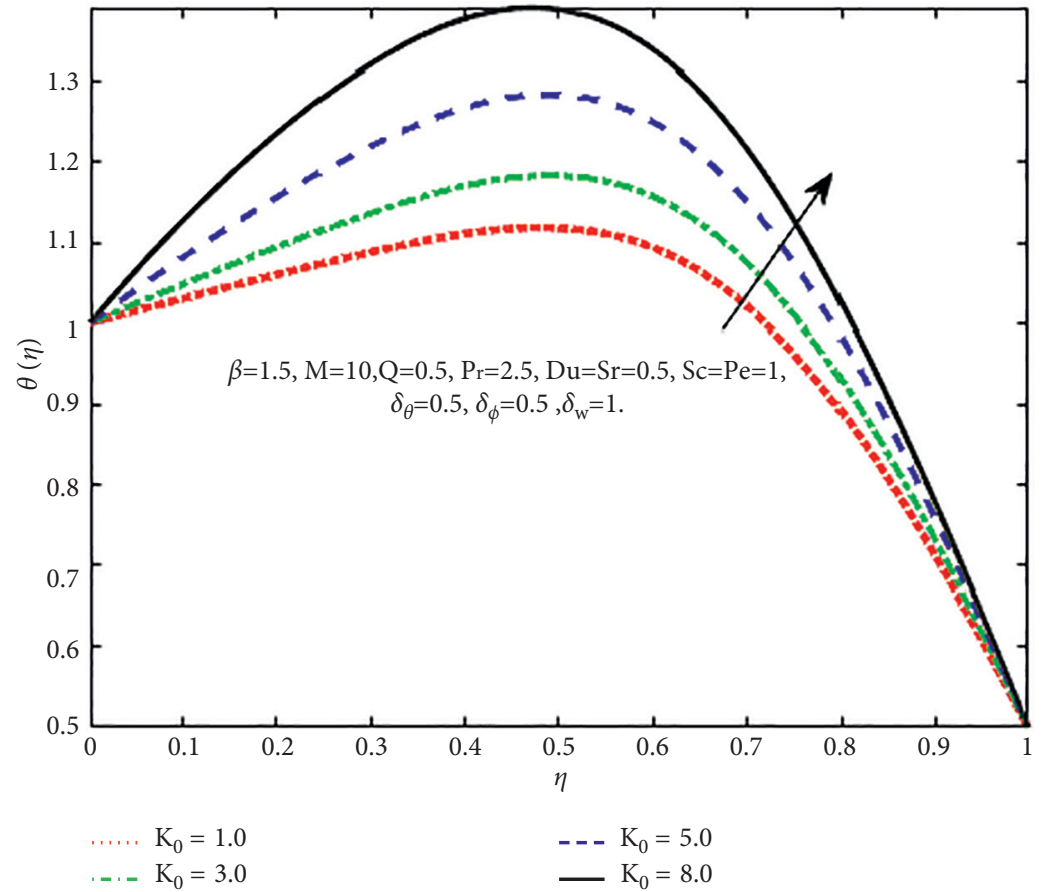

Figure 5: Chemical reaction parameter $\left(\mathbf{K}_{0}\right)$ effect on $\theta(\eta)$.

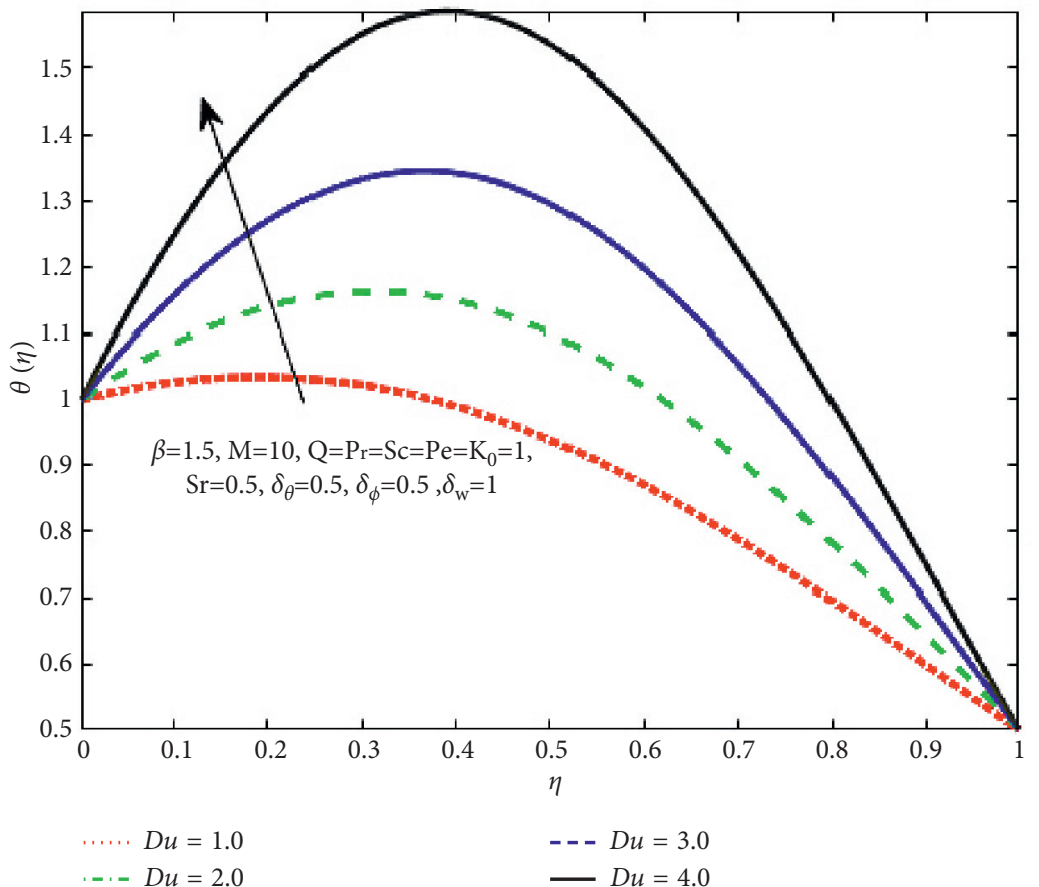

Figure 6: Dufour parameter (Du) effect on $\theta(\eta)$.

indicate increased directional movement of microorganisms, resulting in reduced $w(\eta)$ profile.

Moreover, the impacts of some emerging parameters on the skin friction coefficient number $\left(\mathbf{C}_{\mathbf{f}}\right)$, Nusselt number
$\left(\left(\mathbf{N u}_{\mathbf{x}}\right)\right.$, Sherwood number $(\mathbf{S h})$ and microorganism mass flux $\left(\mathbf{Q}_{\mathbf{x}}\right)$ are described in Tables 1 and 2. Skin friction coefficient is a special parameter in the studies of heat transfer. 


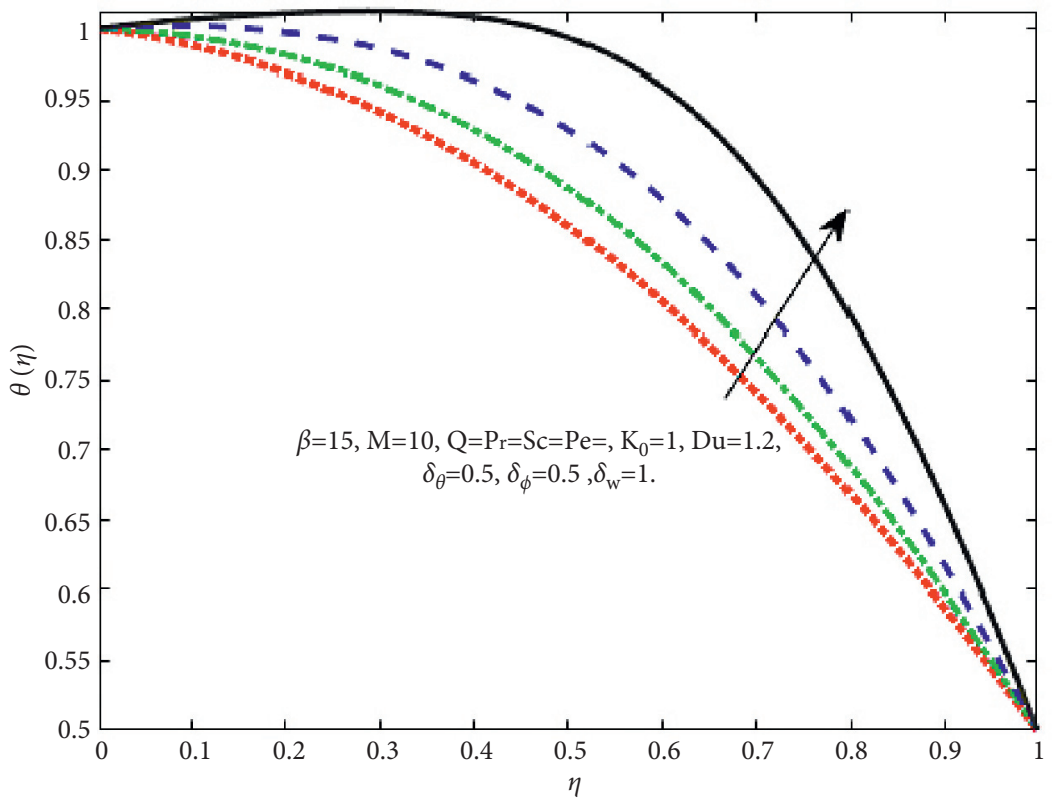

$$
\begin{aligned}
\ldots S & =0.0 & --S r & =0.4 \\
-.-S r & =0.2 & -S r & =0.6
\end{aligned}
$$

Figure 7: Soret parameter (Sr) effect on $\theta(\eta)$.

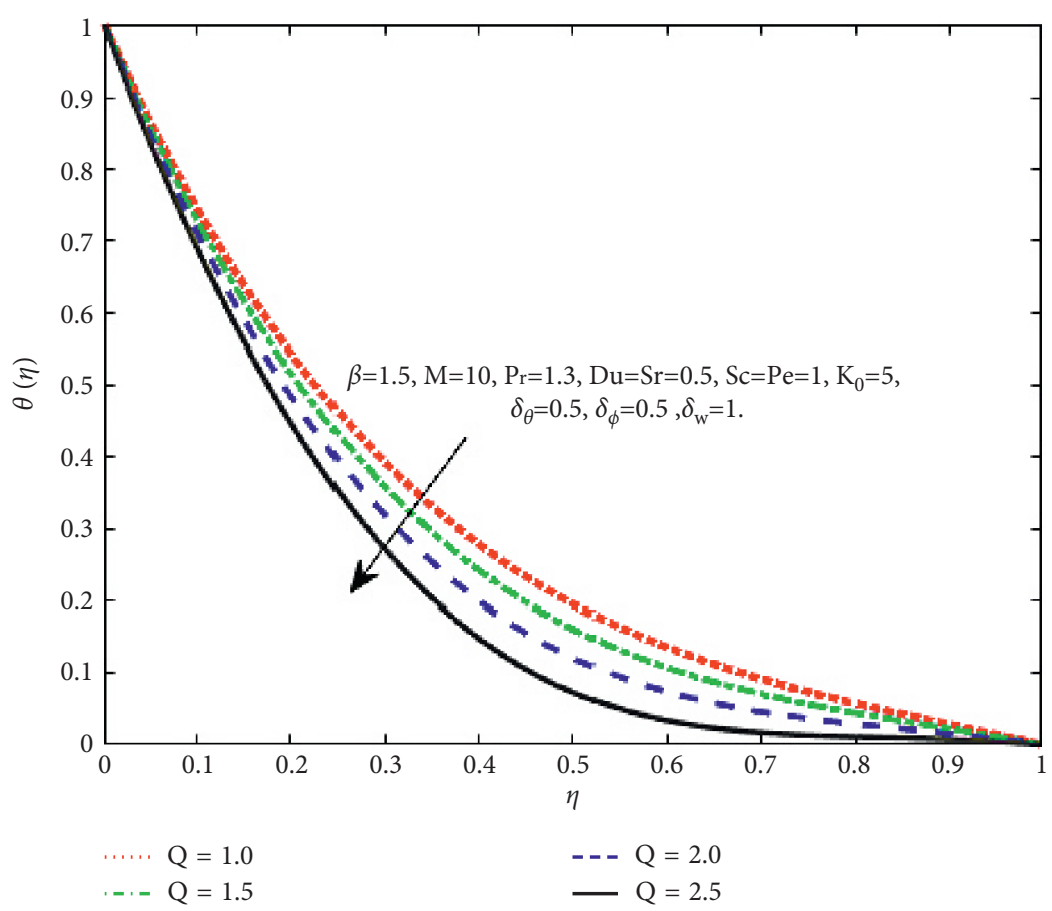

Figure 8: Heat generation parameter (Q) effect on $\phi(\eta)$. 


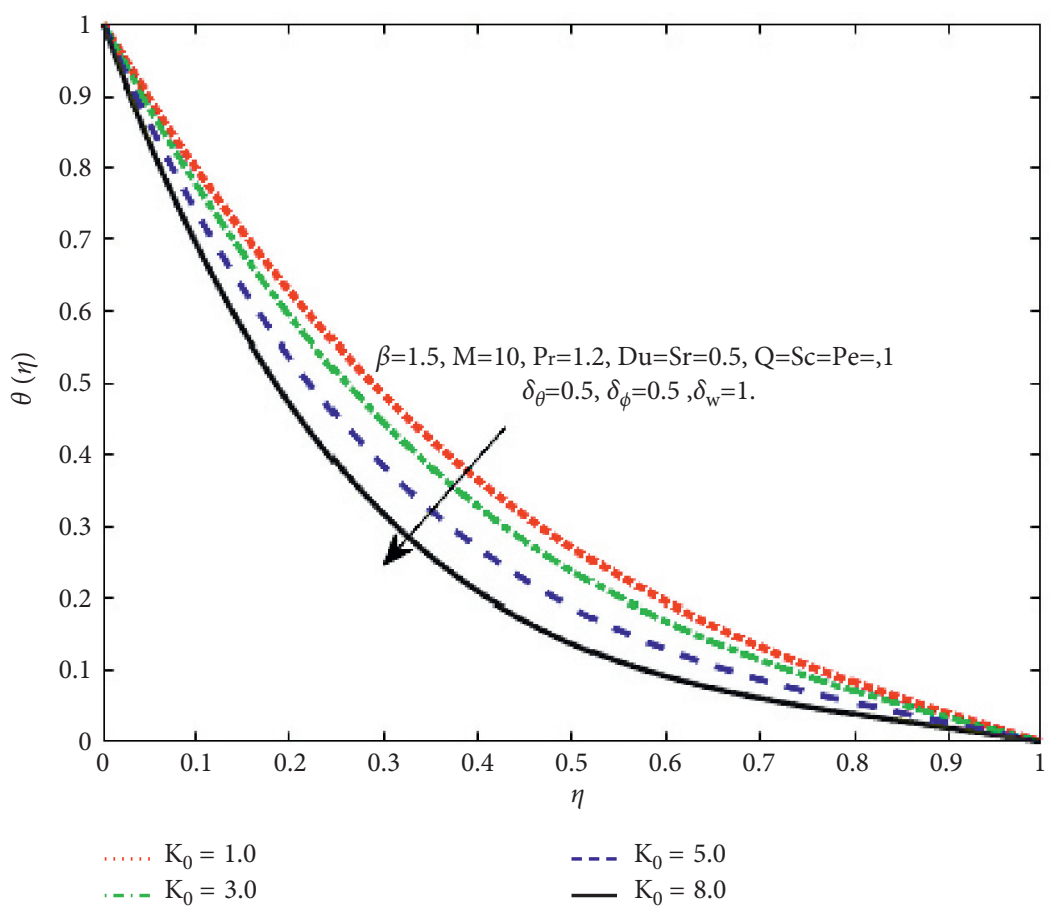

Figure 9: Chemical reaction parameter $\left(\mathbf{K}_{0}\right)$ effect on $\phi(\eta)$.

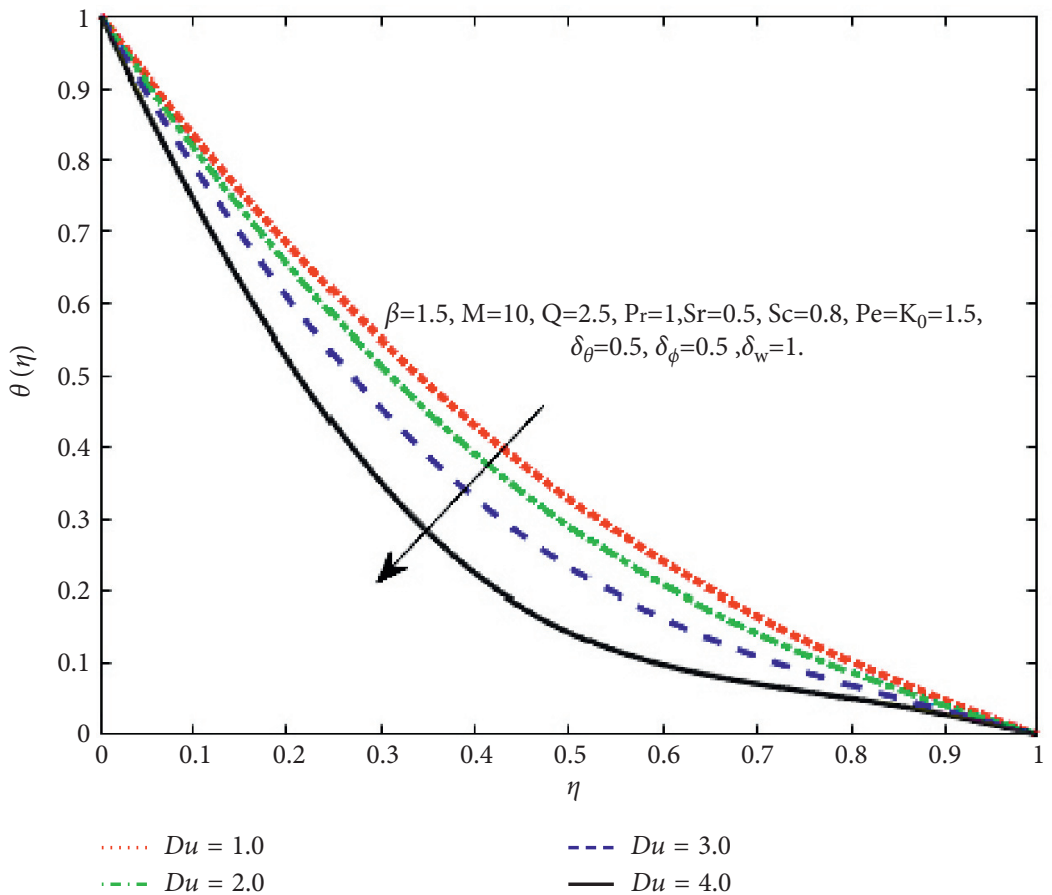

Figure 10: Dufour parameter (Du) effect on $\phi(\eta)$. 


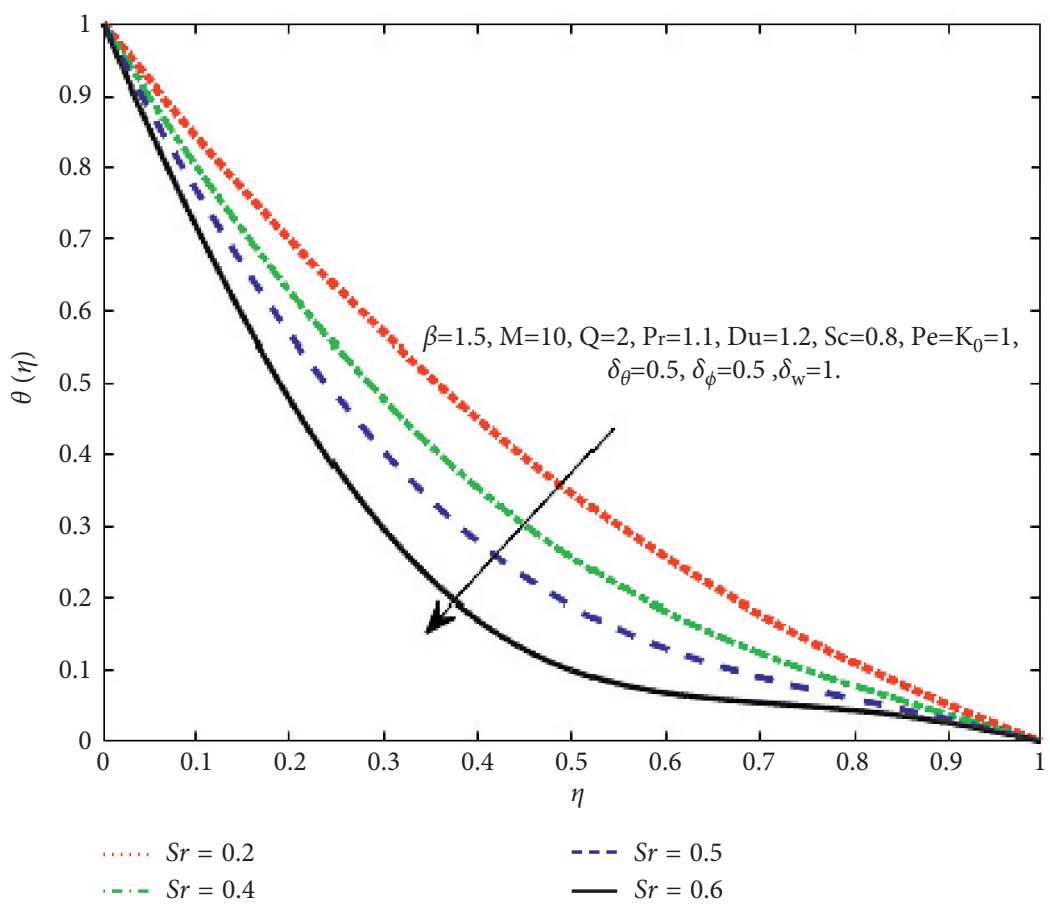

FIGURE 11: Soret parameter (Sr) effect on $\phi(\eta)$.

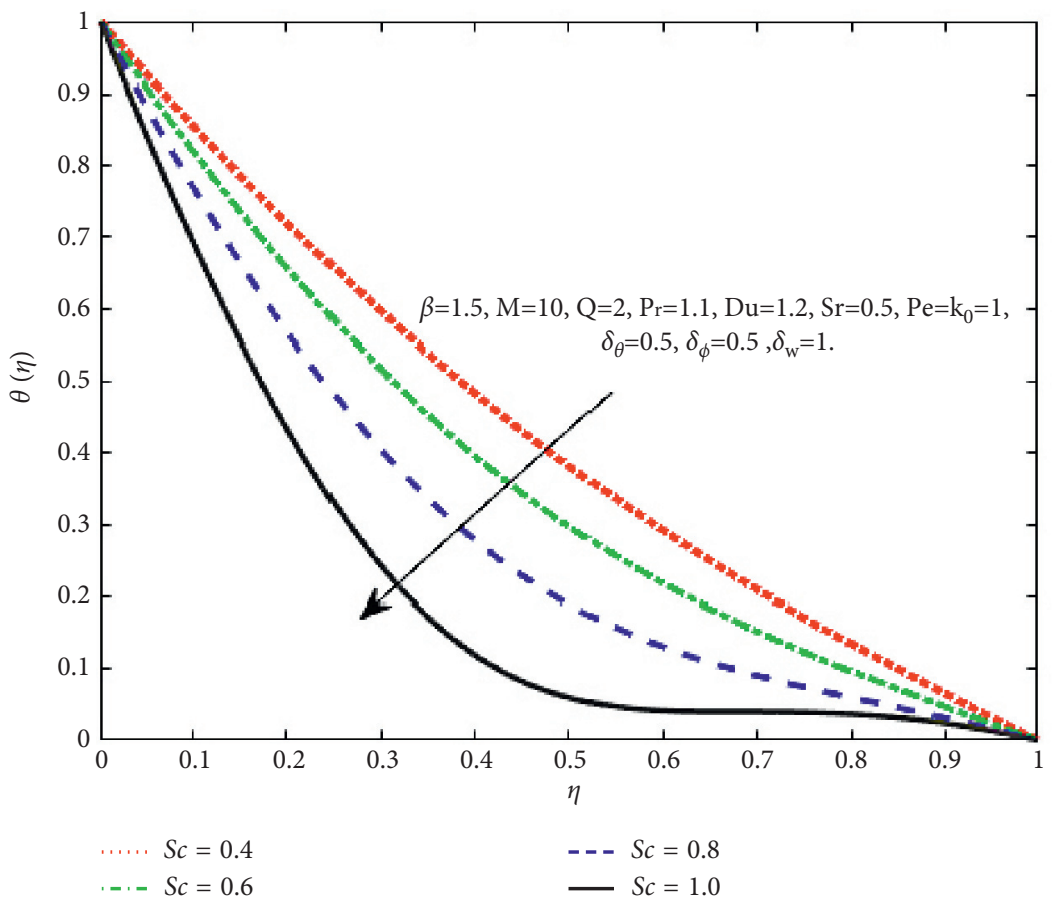

FIgURE 12: Schmidt number (Sc) effect on $\phi(\eta)$. 


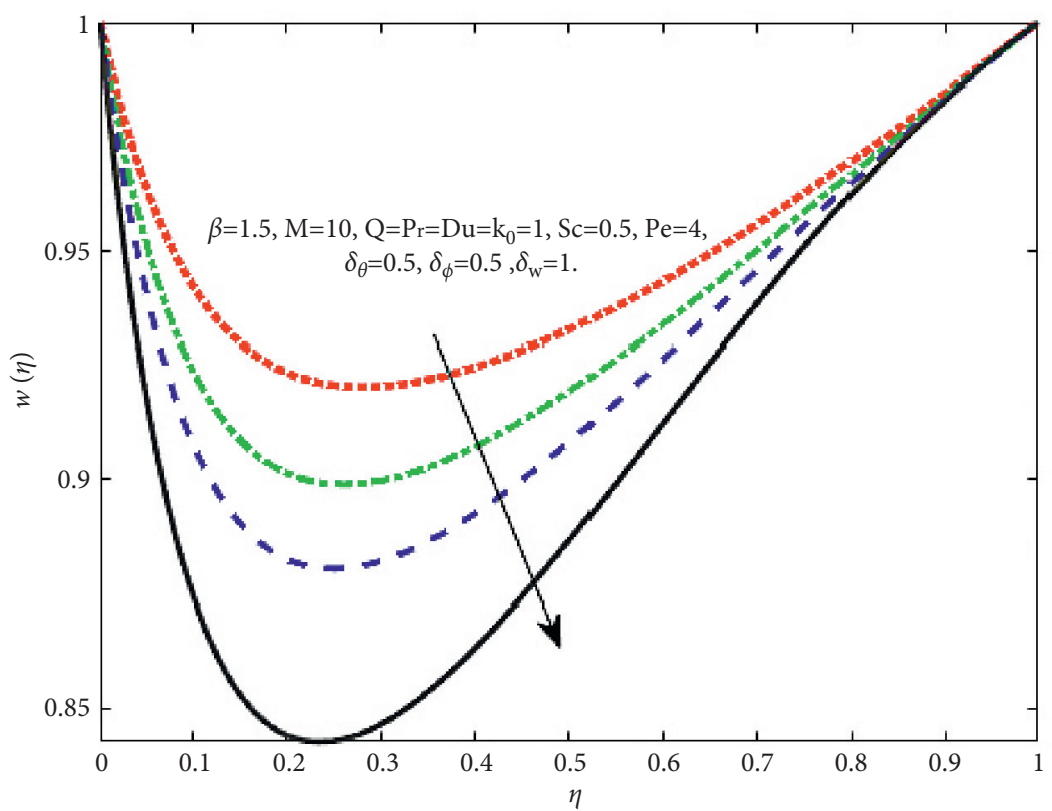

$$
\begin{aligned}
\ldots S & =0.4 & --S r & =0.8 \\
-S S r & =0.6 & -S r & =1.0
\end{aligned}
$$

Figure 13: Soret parameter $(\mathbf{S r})$ effect on $\mathbf{w}(\eta)$.

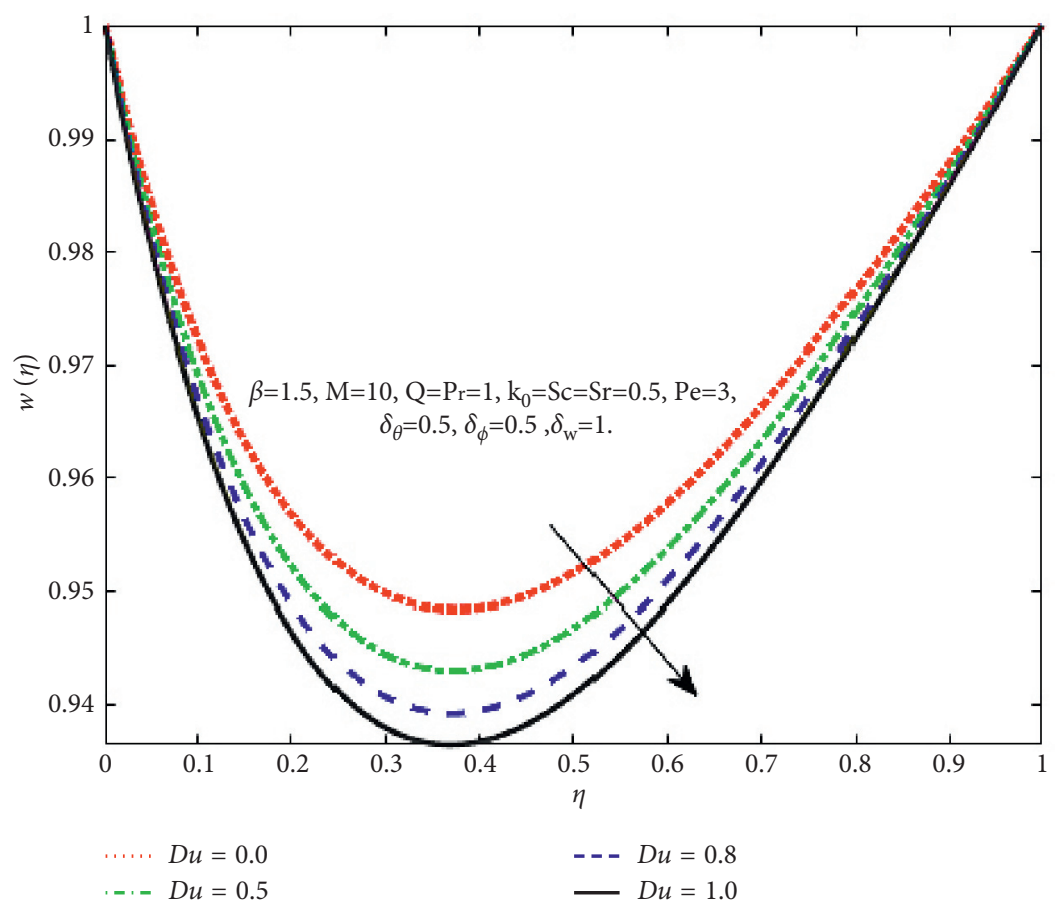

Figure 14: Dufour parameter (Du) effect on $\mathbf{w}(\eta)$. 


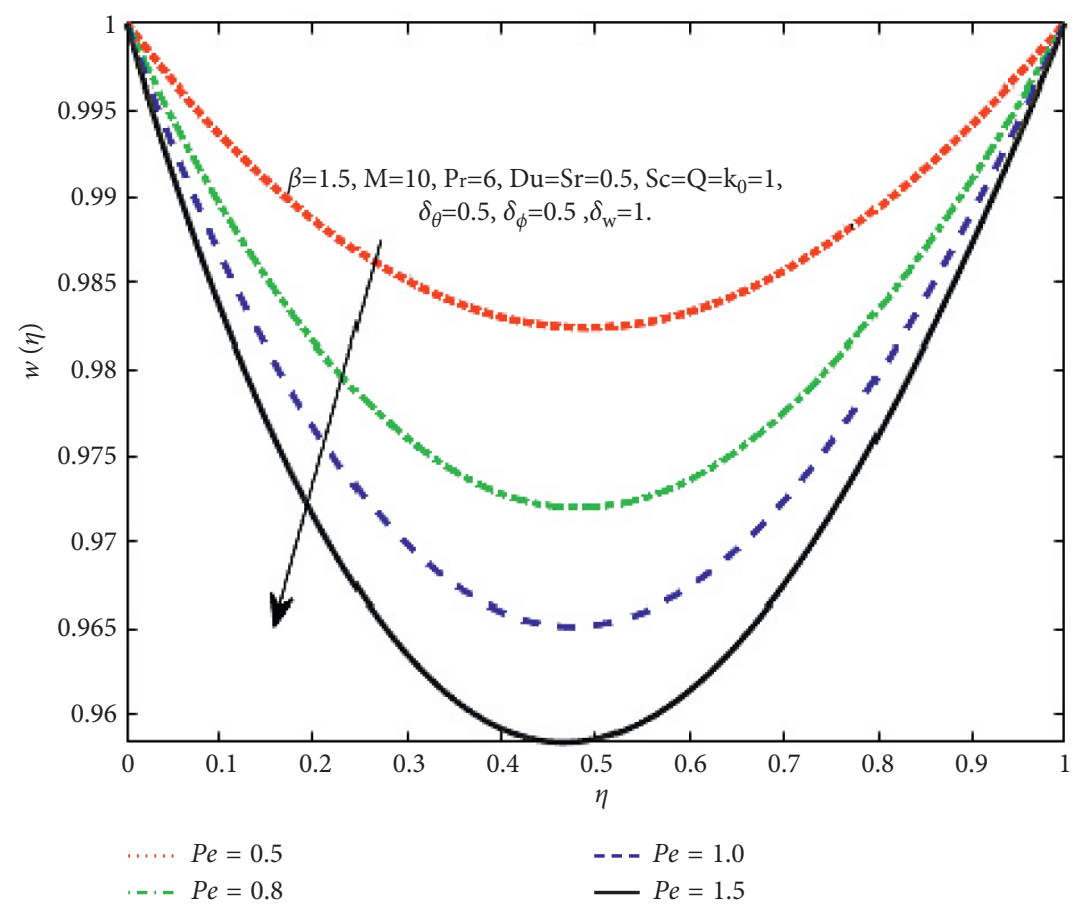

Figure 15: Peclet number (Pe) effect on $\mathbf{w}(\eta)$.

TABLE 1: Skin friction coefficient $\left(\mathbf{C}_{\mathbf{f}}\right)$ for various values of magnetic field $(\mathbf{M})$, and squeezing parameter $(\beta)$ and Nusselt number $\left(\mathbf{N u}_{\mathbf{x}}\right)$ for some distinct values of Prandtl number (Pr) and Dufour number $(\mathrm{Du})$.

\begin{tabular}{|c|c|c|c|c|c|}
\hline $\mathbf{M}$ & $\mathbf{M}$ & $\mathrm{C}_{\mathrm{f}}\left(\mathrm{Re}_{\mathrm{x}}^{-1 / 2}\right)$ & Pr & Du & $\mathrm{Nu}_{\mathrm{x}}\left(\mathrm{Re}_{\mathrm{x}}^{-1 / 2}\right)$ \\
\hline \multirow{3}{*}{0} & 0.5 & -1.170391 & \multirow{3}{*}{0.5} & 0 & -0.6517 \\
\hline & 1 & -1.921779 & & 1 & -1.0567 \\
\hline & 1.5 & 5.183076 & & 1.5 & -3.00877 \\
\hline \multirow{3}{*}{5} & 0.5 & -1.535277 & \multirow{3}{*}{0.7} & 0 & 0.29023 \\
\hline & 1 & 1.799333 & & 1 & 0.00054 \\
\hline & 1.5 & 5.290921 & & 1.5 & -0.22587 \\
\hline \multirow{3}{*}{10} & 0.5 & -1.855036 & \multirow{3}{*}{1} & 0 & 0.20216 \\
\hline & 1 & 1.855036 & & 1 & -0.31621 \\
\hline & 1.5 & 5.411844 & & 1.5 & -0.84232 \\
\hline
\end{tabular}

TABLE 2: Sherwood number (Sh) for several values of Schmidth number $(\mathbf{S c})$ and Soret number $(\mathbf{S r})$ and microorganism mass flux $\left(\mathbf{Q}_{\mathbf{x}}\right)$ for some specific values of Schmidth number $(S c)$ and Peclet number $(\mathbf{P e})$.

\begin{tabular}{lccccc}
\hline Sc & Sr & Sh $\left.\left(\mathbf{R e}_{\mathbf{x}}^{-1 / 2}\right)\right)$ & Sc & Pe & $\mathbf{Q}_{\mathbf{x}}\left(\mathbf{R e}_{\mathbf{x}}^{-1 / 2}\right)$ \\
\hline \multirow{2}{*}{0.5} & 0 & -1.17103 & & 0.5 & -0.16798 \\
& 0.5 & -1.31598 & 0.5 & 1.5 & -0.35603 \\
& 1 & -1.51113 & & 0.5 & -0.56174 \\
1 & 0 & -1.32980 & 1 & 1 & -0.37250 \\
S & 0.5 & -1.70517 & & -0.78721 \\
& 1 & -2.44939 & & 0.5 & -1.28813 \\
1.5 & 0 & -1.47780 & 1.5 & 1.62969 \\
& 0.5 & -2.19863 & & -1.32482 \\
\hline
\end{tabular}




\section{Conclusions}

The Soret and Dufour effects on a two-dimensional unsteady bioconvection squeezing flow incorporating motile gyrotactic microorganisms in a horizontal channel have been examined in the presence of a chemical reaction and a magnetic field. Obtained outcomes are exhibited graphically and in tabulated form. The summarization of results is as given below:

(i) The velocity profile is reduced in the center and slightly increases along the walls of channel.

(ii) Increasing values of squeezing parameter decrease the velocity profile.

(iii) For rising chemical reaction, heat generation/absorption parameters, and Dufour and Soret number values, the temperature profile is increased.

(iv) Chemical reaction parameter is critical for the concentration and motile microorganism's profiles. When the chemical reaction parameter is increased, both profiles diminish.

(v) Fluid concentration drops when the heat generation parameter, Schmidth number, and Dufour and Soret numbers increase.

(vi) The concentration of microorganisms decreases for the rising values of Dufour and Soret, heat generation/absorption parameter, and bioconvection Peclet number.

\section{Nomenclature:}

$V: \quad$ Velocity vector $\left(\mathrm{LT}^{-1}\right)$

B: $\quad$ Magnetic induction $\left(\mathrm{M} \mathrm{A}^{-1} \mathrm{~T}^{-2}\right)$

J: $\quad$ Electric current density $\left(\mathrm{AL}^{-2}\right)$

$\rho: \quad$ Fluid density $\left(\mathrm{ML}^{-3}\right)$

$p$ : $\quad$ Pressure $\left(\mathrm{M} \mathrm{L}^{-1} \mathrm{~T}^{2}\right)$

$v: \quad$ Kinematic viscosity $\left(\mathrm{L}^{2} \mathrm{~T}^{-1}\right)$

T: $\quad$ Temperature (K)

$T_{0}: \quad$ Reference temperature $(\mathrm{K})$

$T_{m}$ : Mean temperature (K)

$\alpha: \quad$ Thermal diffusivity $\left(\mathrm{L}^{2} \mathrm{~T}^{-1}\right)$

$D$ : $\quad$ Mass diffusivity $\left(\mathrm{L}^{2} \mathrm{~T}^{-1}\right)$

$C$ : Chemical reaction

$C_{0}$ : Reference concentrations

$\mathrm{N}$ : Motile micro-organism concentration

$j: \quad$ Flux of microorganisms

$K_{t}$ : Thermal diffusion ratio $\left(\mathrm{L}^{2} \mathrm{~T}^{-1}\right)$

$u$ : Along $x$-axis fluid velocity $\left(\mathrm{LT}^{-1}\right)$

$v$ : Along $y$-axis fluid velocity $\left(\mathrm{LT}^{-1}\right)$

$\widetilde{v}: \quad$ Microorganisms' average swimming velocity $\left(\mathrm{LT}^{-1}\right)$

$D_{n}$ : Microorganisms' diffusion coefficient $\left(\mathrm{L}^{2} \mathrm{~T}^{-1}\right)$

$\eta$ : Dimensionless variable

$f:$ Dimensionless velocity

$\theta: \quad$ Dimensionless temperature

$\phi$ : Nanoparticle's dimensionless concentration

$w$ : Microorganism's dimensionless concentration

$\beta$ : $\quad$ Squeezing parameter

$M$ : Magnetic field number
Pr: Prandtl number

Q: Heat generation parameter

$D u$ : Dufour numbers

Sr: Soret numbers

Sc: Schmidt number

$K_{0}$ : Chemical reaction parameter

$P e: \quad B o$ convection Peclet number

Le: Lewis number.

\section{Data Availability}

The data used to support the findings of this study are included within the article.

\section{Conflicts of Interest}

The authors declare that they have no conflicts of interest.

\section{Acknowledgments}

The authors extend their appreciation to the Deanship of Scientific Research at King Khalid University, Abha, Saudi Arabia, for funding this work through research groups program under grant number RGP.1/248/43.

\section{References}

[1] D. Pal and S. K. Mondal, "MHD nanofluid bioconvection over an exponentially stretching sheet in the presence of gyrotactic microorganisms and thermal radiation," BioNanoScience, vol. 8, no. 1, pp. 272-287, 2018.

[2] A. V. Kuznetsov and A. A. Avramenko, "Effect of small particles on this stability of bioconvection in a suspension of gyrotactic microorganisms in a layer of finite depth," International Communications in Heat and Mass Transfer, vol. 31, no. 1, pp. 1-10, 2004.

[3] W. A. Khan, O. D. Makinde, and Z. H. Khan, "MHD boundary layer flow of a nanofluid containing gyrotactic microorganisms past a vertical plate with Navier slip," International Journal of Heat and Mass Transfer, vol. 74, pp. 285-291, 2014.

[4] L. Tham, R. Nazar, and I. Pop, "Mixed convection flow over a solid sphere embedded in a porous medium filled by a nanofluid containing gyrotactic microorganisms," International Journal of Heat and Mass Transfer, vol. 62, pp. 647-660, 2013.

[5] H. Xu and I. Pop, "Fully developed mixed convection flow in a horizontal channel filled by a nanofluid containing both nanoparticles and gyrotactic microorganisms," European Journal of Mechanics - B: Fluids, vol. 46, pp. 37-45, 2014.

[6] A. Raees, H. Xu, and S.-J. Liao, "Unsteady mixed nanobioconvection flow in a horizontal channel with its upper plate expanding or contracting," International Journal of Heat and Mass Transfer, vol. 86, pp. 174-182, 2015.

[7] S. Mosayebidorcheh, M. A. Tahavori, T. Mosayebidorcheh, and D. D. Ganji, "Analysis of nano-bioconvection flow containing both nanoparticles and gyrotactic microorganisms in a horizontal channel using modified least square method (MLSM)," Journal of Molecular Liquids, vol. 227, pp. 356-365, 2017.

[8] B. Shen, L. Zheng, C. Zhang, and X. Zhang, "Bioconvection heat transfer of a nanofluid over a stretching sheet with 
velocity slip and temperature jump," Thermal Science, vol. 21, no. 6, pp. 2347-2356, 2017.

[9] R. Kumar, S. Sood, S. A. Shehzad, and M. Sheikholeslami, "Numerical modeling of time-dependent bio-convective stagnation flow of a nanofluid in slip regime," Results in Physics, vol. 7, pp. 3325-3332, 2017.

[10] Q. Zhao, H. Xu, and L. Tao, "Unsteady bioconvection squeezing flow in a horizontal channel with chemical reaction and magnetic field effects," Mathematical Problems in Engineering, vol. 2017, Article ID 2541413, 2017.

[11] N. Tarakaramu and P. V. Satya Narayana, "Chemical reaction effects on bio-convection nanofluid flow between two parallel plates in rotating system with variable viscosity: a numerical study," Journal of Applied and Computational Mechanics, vol. 5, no. 4, pp. 791-803, 2019.

[12] H. Waqas, S. U. Khan, M. Hassan, M. M. Bhatti, and M. Imran, "Analysis on the bioconvection flow of modified second-grade nanofluid containing gyrotactic microorganisms and nanoparticles," Journal of Molecular Liquids, vol. 291, Article ID 111231, 2019.

[13] A. M. Rashad and H. A. Nabwey, "Gyrotactic mixed bioconvection flow of a nanofluid past a circular cylinder with convective boundary condition," Journal of the Taiwan Institute of Chemical Engineers, vol. 99, pp. 9-17, 2019.

[14] N. Shukla, P. Rana, S. Kuharat, and O. A. Bég, "Non-similar radiative bioconvection nanofluid flow under oblique magnetic field with entropy generation," Journal of Applied and Computational Mechanics, vol. 8, no. 1, pp. 206-218, 2020.

[15] C.-Y. Cheng, "Soret and Dufour effects on heat and mass transfer by natural convection from a vertical truncated cone in a fluid-saturated porous medium with variable wall temperature and concentration," International Communications in Heat and Mass Transfer, vol. 37, no. 8, pp. 1031-1035, 2010.

[16] T. Hayat, M. Mustafa, and I. Pop, "Heat and mass transfer for Soret and Dufour's effect on mixed convection boundary layer flow over a stretching vertical surface in a porous medium filled with a viscoelastic fluid," Communications in Nonlinear Science and Numerical Simulation, vol. 15, no. 5, pp. 1183-1196, 2010.

[17] T. Hayat and M. Nawaz, "Soret and Dufour effects on the mixed convection flow of a second grade fluid subject to Hall and ion-slip currents," International Journal for Numerical Methods in Fluids, vol. 67, no. 9, pp. 1073-1099, 2011.

[18] B. K. Sharma, K. Yadav, N. K. Mishra, and R. C. Chaudhary, "Soret and Dufour Effects on Unsteady MHD Mixed Convection Flow Past a Radiative Vertical Porous Plate Embedded in a Porous Medium with Chemical Reaction," Applied Mathematics, vol. 03, no. 07, 2012.

[19] T. Hayat, A. Safdar, M. Awais, and S. Mesloub, "Soret and Dufour effects for three-dimensional flow in a viscoelastic fluid over a stretching surface," International Journal of Heat and Mass Transfer, vol. 55, no. 7-8, pp. 2129-2136, 2012.

[20] M. B. K. Moorthy, T. Kannan, and K. Senthilvadivu, "Soret and Dufour effects on natural convection heat and mass transfer flow past a horizontal surface in a porous medium with variable viscosity," WSEAS Transactions on Heat and Mass Transfer, vol. 8, no. 4, pp. 74-83, 2013.

[21] S. R. Sheri and R. Srinivasa Raju, "Soret effect on unsteady MHD free convective flow past a semi-infinite vertical plate in the presence of viscous dissipation," International Journal for Computational Methods in Engineering Science and Mechanics, vol. 16, no. 2, pp. 132-141, 2015.

[22] A. Majeed, T. Javed, and A. Ghaffari, "Numerical investigation on flow of second grade fluid due to stretching cylinder with
Soret and Dufour effects," Journal of Molecular Liquids, vol. 221, pp. 878-884, 2016.

[23] Q. Liu, X.-B. Feng, X.-T. Xu, and Y.-L. He, "Multiple-relaxation-time lattice Boltzmann model for double-diffusive convection with Dufour and Soret effects," International Journal of Heat and Mass Transfer, vol. 139, pp. 713-719, 2019.

[24] H. Sardar, L. Ahmad, M. Khan, and A. S. Alshomrani, "Investigation of mixed convection flow of Carreau nanofluid over a wedge in the presence of Soret and Dufour effects," International Journal of Heat and Mass Transfer, vol. 137, pp. 809-822, 2019.

[25] M. Bilal Ashraf, T. Hayat, S. A. Shehzad, and B. Ahmed, "Thermophoresis and MHD mixed convection three-dimensional flow of viscoelastic fluid with Soret and Dufour effects," Neural Computing \& Applications, vol. 31, no. 1, pp. 249-261, 2019.

[26] N. Jiang, E. Studer, and B. Podvin, "Physical modeling of simultaneous heat and mass transfer: species interdiffusion, Soret effect and Dufour effect," International Journal of Heat and Mass Transfer, vol. 156, Article ID 119758, 2020.

[27] A. Hafeez, M. Khan, and J. Ahmed, "Oldroyd-B fluid flow over a rotating disk subject to Soret-Dufour effects and thermophoresis particle deposition," Proceedings of the Institution of Mechanical Engineers - Part C: Journal of Mechanical Engineering Science, vol. 235, no. 13, Article ID 0954406220946075, 2020.

[28] M. K. Nayak, "MHD 3D flow and heat transfer analysis of nanofluid by shrinking surface inspired by thermal radiation and viscous dissipation," International Journal of Mechanical Sciences, vol. 124-125, pp. 185-193, 2017.

[29] B. Ramandevi, J. V. R. Reddy, V. Sugunamma, and N. Sandeep, "Combined influence of viscous dissipation and non-uniform heat source/sink on MHD non-Newtonian fluid flow with Cattaneo-Christov heat flux," Alexandria Engineering Journal, vol. 57, no. 2, pp. 1009-1018, 2018.

[30] U. Farooq, D. Lu, S. Munir, M. Ramzan, M. Suleman, and S. Hussain, "MHD flow of Maxwell fluid with nanomaterials due to an exponentially stretching surface," Scientific Reports, vol. 9, no. 1, pp. 7312-7411, 2019.

[31] B. Mahanthesh, B. J. Gireesha, I. L. Animasaun, T. Muhammad, and N. S. Shashikumar, "MHD flow of SWCNT and MWCNT nanoliquids past a rotating stretchable disk with thermal and exponential space dependent heat source," Physica Scripta, vol. 94, no. 8, Article ID 085214, 2019.

[32] H. R. Patel and R. Singh, "Thermophoresis, Brownian motion and non-linear thermal radiation effects on mixed convection MHD micropolar fluid flow due to nonlinear stretched sheet in porous medium with viscous dissipation, joule heating and convective boundary condition," International Communications in Heat and Mass Transfer, vol. 107, pp. 68-92, 2019.

[33] E. H. Aly and I. Pop, "MHD flow and heat transfer over a permeable stretching/shrinking sheet in a hybrid nanofluid with a convective boundary condition," International Journal of Numerical Methods for Heat and Fluid Flow, 2019.

[34] I. Rashid, M. Sagheer, and S. Hussain, "Entropy formation analysis of MHD boundary layer flow of nanofluid over a porous shrinking wall," Physica A: Statistical Mechanics and Its Applications, vol. 536, Article ID 122608, 2019.

[35] I. Waini, A. Ishak, and I. Pop, "MHD flow and heat transfer of a hybrid nanofluid past a permeable stretching/shrinking wedge," Applied Mathematics and Mechanics, vol. 41, no. 3, pp. 507-520, 2020. 
[36] S. M. R. S. Naqvi, T. Muhammad, S. Saleem, and H. M. Kim, "Significance of non-uniform heat generation/absorption in hydromagnetic flow of nanofluid due to stretching/shrinking disk," Physica A: Statistical Mechanics and Its Applications, vol. 553, Article ID 123970, 2020.

[37] A. Raees, U. Farooq, M. Hussain, W. A. Khan, and F. B. Farooq, "Non-similar mixed convection analysis for magnetic flow of second-grade nanofluid over a vertically stretching sheet," Communications in Theoretical Physics, vol. 73, no. 6, Article ID 065801, 2021.

[38] R. Rizwana, A. Hussain, and S. Nadeem, "Mix convection non- boundary layer flow of unsteady MHD oblique stagnation point flow of nanofluid," International Communications in Heat and Mass Transfer, vol. 124, Article ID 105285, 2021.

[39] S. Liao, Homotopy Analysis Method in Nonlinear Differential Equations, pp. 153-165, Higher education press, Beijing, China, 2012. 